\title{
Astragalus polysaccharide has a protective effect on hematopoiesis in an irradiated mouse model and decreases apoptosis in megakaryocytes
}

\author{
LIANG LI $^{1 *}$, WANHUA XU ${ }^{2 *}$, CHENJU YI $^{1}$, YUCAI CHENG ${ }^{1}$, HONGWU XIN ${ }^{3,4}$, HONGMAN XUE ${ }^{1}$, \\ CHI-KONG LI ${ }^{5}$, XIAOYI FANG ${ }^{1}$, LIUMING YANG ${ }^{3}$, CHUN CHEN $^{1}$ and MO YANG ${ }^{1-3}$ \\ ${ }^{1}$ Research Center of The Seventh Affiliated Hospital, Sun Yat-sen University, Shenzhen, Guangdong 518107; \\ ${ }^{2}$ Department of Hematology, Nanfang Hospital, Southern Medical University, Guangzhou, Guangdong 510515; ${ }^{3}$ Department \\ of Medicine, Lianjiang People's Hospital, Lianjiang, Guangdong 524400; ${ }^{4}$ Laboratory of Oncology, Center for Molecular \\ Medicine, School of Basic Medicine, Health Science Center, Yangtze University, Jingzhou, Hubei 434023; ${ }^{5}$ Department \\ of Pediatrics, The Chinese University of Hong Kong, Prince of Wales Hospital, Hong Kong 999077, SAR, P.R. China
}

Received May 12, 2020; Accepted September 24, 2020

DOI: $10.3892 / \mathrm{mmr} .2020 .11653$

\begin{abstract}
Huangqi, the dried root of Radix Astragali, is an essential herb in Traditional Chinese Medicine and has been used to promote hematopoiesis for centuries. Astragalus polysaccharide (ASPS), the bioactive compound of Huangqi, serves a crucial role in hematopoiesis. The aim of the present study was to investigate the hematopoietic effects, in particular the thrombopoietic effects, and the molecular mechanisms of ASPS using an irradiation-induced myelosuppressive mouse model. Colony-forming unit assays, flow cytometric analysis of apoptosis, ELISAs, Giemsa staining and western blotting were performed to determine the hematopoietic and anti-apoptotic effects of ASPS. The results demonstrated that ASPS enhanced the recovery of red blood cells at day 21 following treatment, as well as platelets and white blood cells at day 14. In addition, ASPS promoted colony formation in all lineages (megakaryocytes, granulocyte monocytes, erythroid cells and fibroblasts). The morphological study of the bone marrow demonstrated that tri-lineage hematopoiesis was preserved in the ASPSand thrombopoietin (TPO)-treated groups compared with the control group. The overall cellularity (mean total cell count/area) of the ASPS-treated group was similar to that of the TPO-treated group. Additionally, in vitro experiments
\end{abstract}

Correspondence to: Professor Mo Yang or Professor Chun Chen, Research Center of The Seventh Affiliated Hospital, Sun Yat-sen University, 628 Zhenyuan Road, Guangming, Shenzhen, Guangdong 518107, P.R. China

E-mail: yangm1091@126.com

E-mail: chenchun69@126.com

*Contributed equally

Key words: apoptosis, astragalus polysaccharide, hematopoiesis, megakaryocytes, thrombopoiesis indicated that treatment with $100 \mu \mathrm{g} / \mathrm{ml}$ ASPS exhibited the maximum effect on colony formation. ASPS attenuated cell apoptosis in megakaryocytic cells via inhibiting the mitochondrial caspase-3 signaling pathway. In conclusion, ASPS promoted hematopoiesis in irradiated myelosuppressive mice possibly via enhancing hematopoietic stem/progenitor cell proliferation and inhibiting megakaryocytes apoptosis.

\section{Introduction}

Patients with cancer, such as leukemia, exhibit bone marrow suppression, including thrombocytopenia, which is characterized by subnormal levels of blood platelets following chemotherapy or radiotherapy (1). Platelet transfusion (2) and thrombopoietin (TPO) treatment (3) are commonly used for the management of thrombocytopenia. However, aggravation of thrombocytopenia may be induced due to the subsequent formation of anti-platelet and anti-TPO antibodies $(3,4)$. Furthermore, repeated platelet transfusions have been associated with increased risk of blood infections (5). It has also been reported that treatment with recombinant human TPO (rhTPO) therapy results in the formation of TPO antibodies, leading to severe thrombocytopenia $(6,7)$. Therefore, there is a need for the development of safer and more effective therapies for thrombocytopenia.

In ancient China, a variety of Chinese herbal decoctions have been used to treat blood disorders. For instance, Danggui Buxue Tang (DBT), a renowned formulation that has been used for $>800$ years, consists of two herbs, namely Danggui (extracted from Radix Angelica Sinensis) and Huangqi (extracted from Radix Astragali) $(8,9)$. Our previous studies have confirmed the hematopoietic and thrombopoietic effects of DBT and polysaccharides, extracted from the Angelica Sinensis root, on irradiated mice $(10,11)$. These studies indicated that treatment with DBT significantly increased the recovery of megakaryocytes, as well as enhanced platelet recovery and the number of colony forming unit-megakaryocytes (CFU-MK) in vivo $(10,11)$. Furthermore, the polysaccharide fraction of 
Angelica Sinensis increased not only the recovery of platelets, other blood cells and their progenitor cells, but also the number of CFUs (10).

However, the role of Radix Astragali, another major component of DBT, in hematopoiesis and thrombopoiesis is yet to be fully elucidated. Several compounds have been identified in Radix Astragali, including various polysaccharides, glycosides, alkaloids, volatile oils and organic acids (12). Among them, astragalus polysaccharide (ASPS) from the primary constituent of Radix Astragali is considered the most important bioactive component, as it is hypothesized that polysaccharides are the main components in plants that promote hematopoiesis (13). In addition, our previous study also confirmed that angelica polysaccharides promoted hematopoiesis (10). Therefore, the present study aimed to investigate the effects and mechanisms of ASPS on hematopoiesis and megakaryocytes.

\section{Materials and methods}

Ethics statement. The Animal Research Welfare Committee of Southern Medical University approved the present experimental protocol. The principles of the National Institutes of Health Guidelines for Laboratory Animals (14) were followed during the entire course of the experiments.

Preparation of herbal materials. Radix Astragali, provided and identified by the Institute of Chinese Medicine, The Chinese University of Hong Kong, is currently deposited in the Molecular Chinese Medicine Laboratory, University of Hong Kong (voucher no. mcm-010) (10,11).

For the preparation of ASPS (15), Radix Astragali was sliced, boiled in water and the extract was filtered three times. The three filtrates were pooled and concentrated to $30 \mathrm{ml}$ for $1 \mathrm{~h}$ in a rotary evaporator at $70^{\circ} \mathrm{C}$. After removing the protein content using the Sevag method (16), the solution was precipitated with $95 \%$ ethanol and collected via centrifugation at 2,500 x g for $10 \mathrm{~min}$ at $4^{\circ} \mathrm{C}$. Subsequently, the pellet was dissolved in distilled water, dialyzed for $48 \mathrm{~h}$ and then lyophilized, to eventually obtain the white, powdery polysaccharide.

Animals. A total of 18 male BALB/c mice (age, 7-8 weeks; weight, 18-20 g) were purchased from Charles River Laboratories Japan, Inc., and given free access to food and water. Mice were housed in $400 \times 332 \times 286-\mathrm{mm}$ cages (6 mice/cage) in temperature-controlled rooms (temperature, $24 \pm 2^{\circ} \mathrm{C}$; humidity, $50 \pm 5 \%$ ) under a 12-h light/dark cycle. Mice were monitored twice daily for their health status.

Radiation-induced hemocytopenia/thrombocytopenia mouse model. Mice were randomly divided into the following four groups: i) Control (radiation-induced, saline treated) group; ii) ASPS-treated group; iii) rhTPO (TPO)-treated group; and iv) normal (untreated) group. The hemocytopenia/thrombocytopenia model was established using $4 \mathrm{~Gy}$ irradiation from a ${ }^{137}$ Cs source (Gammacell-1000 Elite Irradiator; Nordion, Inc.) as previously described $(10,11,17)$. Mice underwent intraperitoneal administration of ASPS $(12.5 \mathrm{mg} /$ day $)(10,11)$ or rhTPO (1 $\mu \mathrm{g} / \mathrm{kg} / \mathrm{day}$; 3SBio, Inc.) daily for 21 days starting from the day following radiotherapy. Control mice were administered with an intraperitoneal injection of saline $(1.25 \mathrm{ml})$.

Bone marrow samples were frozen in cyomolds at $-74^{\circ} \mathrm{C}$ for $2 \mathrm{~h}$, cut into $5-\mu \mathrm{m}$ thick sections and fixed in $95 \%$ ethyl alcohol at $4^{\circ} \mathrm{C}$ for $5 \mathrm{~min}$, and then stained with Giemsa for $25 \mathrm{~min}$ at room temperature. A total of 25 random high-power fields from each bone marrow sample were chosen and visualized using a light microscope (magnification, $\mathrm{x} 40$ ) to determine the mean total cell count (MTC) (18). Additionally, the mean cell counts of the erythroid, granulocytic and megakaryocytic cell lineages were recorded.

Blood and bone marrow collection. On days 0, 7, 14 and 21 after irradiation, $0.1 \mathrm{ml}$ peripheral blood samples were obtained via tail veins, following administration of isoflurane (3.5\% induction for $4 \mathrm{~min}$ and $1.5 \%$ maintenance) anesthesia. Blood cell counts were analyzed using a ProCyte Dx Hematology analyzer (IDEXX Laboratories, Inc.). The body weight of all mice was also recorded in order to evaluate the general effects of ASPS or other treatments on well-being of animals. All mice were euthanized via cervical dislocation on day 21 .

For the CFU assays, bone marrow cells were harvested from the proximal femur by inserting a needle and forcing cell culture medium (IMDM; Gibco; Thermo Fisher Scientific, Inc.) supplemented with 10\% FCS (HyClone; Cytiva) through the bone shaft. Murine bone marrow cells ( $2 \times 10^{5}$ nucleated cells) were cultured in $35-\mathrm{mm}$ Petri dishes using the plasma clot culture method $(10,11,19)$. The medium comprised $1 \%$ deionized BSA (Sigma-Aldrich; Merck KGaA), $0.34 \mathrm{mg} \mathrm{CaCl}, 10 \%$ citrated bovine plasma (Sigma-Aldrich; Merck KGaA), 100 units penicillin (Gibco; Thermo Fisher Scientific, Inc.), $50 \mu \mathrm{g}$ streptomycin (Gibco; Thermo Fisher Scientifc, Inc.), $0.1 \mathrm{mM} \beta$-mercaptoethanol (Sigma-Aldrich; Merck KGaA), $3 \mathrm{IU} / \mathrm{ml}$ erythropoietin (Cilag AG), $10 \mathrm{ng} / \mathrm{ml}$ IL-3 (PeproTech, Inc.), $50 \mathrm{ng} / \mathrm{ml}$ stem cell factor (PeproTech, Inc.) and IMDM in a total volume of $1 \mathrm{ml}$. The dishes were incubated at $37^{\circ} \mathrm{C}$ in a fully humidified atmosphere with $5 \% \mathrm{CO}_{2}$.

$C F U-M K$ assay. Murine bone marrow was exposed by cutting the ends of the femurs and was extruded by inserting a needle and forcing cell culture medium with 10\% FCS (HyClone; Cytiva) through the bone shaft. Murine bone marrow cells ( $2 \times 10^{5}$ cells) were cultured in $35-\mathrm{mm}$ Petri dishes using the plasma clot culture method $(10,18)$. The system contained $1 \%$ deionized BSA, $0.34 \mathrm{mg} \mathrm{CaCl}_{2}, 10 \%$ citrated bovine plasma (all from Sigma-Aldrich; Merck KGaA), $100 \mu \mathrm{g}$ penicillin and $50 \mu \mathrm{g}$ streptomycin in Iscove's modified Dulbecco's medium (IMDM; Thermo Fisher Scientific, Inc.) supplemented with different concentrations of ASPS $(0,50,100$ or $200 \mu \mathrm{g} / \mathrm{ml})$, TPO $(50 \mathrm{ng} / \mathrm{ml})$ in a total volume of $1 \mathrm{ml}$. Cells were incubated at $37^{\circ} \mathrm{C}$ and $5 \% \mathrm{CO}_{2}$ atmosphere for 7 days. Following incubation for 7 days, the number of CFU-MK-derived colonies was counted using the acetylcholine esterase staining method (incubation for $20-30 \mathrm{~min}$ at $\left.37^{\circ} \mathrm{C}\right)(10,11)$. Subsequently, cells were stained with hematoxylin for $5 \mathrm{~min}$ at room temperature to count the CFU-granulocyte macrophage (CFU-GM)-derived colonies using a light microscope (magnification, x10). A CFU-MK colony was defined as a cluster of $\geq 3$ acetylcholine 
esterase-positive cells, while a CFU-GM colony consisted of a cluster of $\geq 40$ cells (10).

Murine bone marrow CFU-fibroblast (CFU-F) assay. A CFU-F assay was performed as previously described $(10,18)$. Briefly, murine bone marrow cells $\left(1 \times 10^{6}\right.$ cells) from different groups were resuspended in $2 \mathrm{ml}$ IMDM supplemented with $10 \%$ FCS in the presence of ASPS $(100 \mu \mathrm{g} / \mathrm{ml})$ or TPO $(50 \mathrm{ng} / \mathrm{ml})$, and incubated at $37^{\circ} \mathrm{C}$ in a fully humidified atmosphere with $5 \% \mathrm{CO}_{2}$ for 9 days. All procedures were performed in triplicate. A CFU-F colony was defined as an aggregate containing $\geq 20$ fibroblasts (18). Subsequently, adherent cells were stained with Giemsa for $25 \mathrm{~min}$ at room temperature, and the CFU-F colonies were counted under an Olympus CKX53 inverted light microscope (Olympus Corporation) at x10 magnification.

CFU-GM, burst-forming unit/CFU-erythroid (BFU/CFU-E) and $C F U$-granulocyte, erythroid, monocyte and megakaryocyte (GEMM) assays. To further evaluate the effects of ASPS $(100 \mu \mathrm{g} / \mathrm{ml})$ on hematologic progenitor cells, CFU-GM, BFU/CFU-E and CFU-GEMM assays were performed $(10,11,20)$. Briefly, murine bone marrow cells $\left(2 \times 10^{5}\right.$ cells $\left./ \mathrm{ml}\right)$ were cultured in culture medium supplemented with $1 \%$ methylcellulose (Sigma-Aldrich; Merck KGaA), 30\% FCS, 1\% BSA, $0.1 \mathrm{mM} \beta$-mercaptoethanol (Sigma-Aldrich; Merck KGaA), $3 \mathrm{IU} / \mathrm{ml}$ erythropoietin (Cilag AG), $10 \mathrm{ng} / \mathrm{ml} \mathrm{IL-3} \mathrm{and} 50 \mathrm{ng} / \mathrm{ml}$ stem cell factor (PeproTech, Inc.). Murine bone marrow cells ( $2 \times 10^{5}$ cells $\left./ \mathrm{ml}\right)$ were seeded into $35-\mathrm{mm}$ Petri dishes in triplicate and incubated for 7 days. Finally, colonies were counted blindly using a light microscope (magnification, x10) by two investigators $(10,11)$.

ELISA assay for TPO. Briefly, $0.1 \mathrm{ml}$ murine peripheral blood samples were centrifuged (400 x g; room temperature; $10 \mathrm{~min}$ ) to obtain the plasma. TPO levels were measured using an ELISA kit (R\&D Systems, Inc.; cat. MTP00), according to the manufacturer's instructions. The optical density of each well was measured at $450 \mathrm{~nm}$ using a microplate reader (BioTek Instruments, Inc.).

Annexin V, caspase-3 and 5,5,6,6-tetrachloro-1,1,3,3-Tetraethylbenzimidazolcarbocyanine iodide (JC-1) analysis via flow cytometry. Cells were randomly divided into normal (untreated), control (serum-free, cytokine and serum depleted), TPO-treated, ASPS-treated, LY294002-treated and ASPS + LY294002-treated groups. Flow cytometric analysis was performed as described previously. Briefly, the megakaryoblastic cell line M-07e (American Type Culture Collection) was maintained in IMDM supplemented with granulocyte-macrophage colony-stimulating factor $(20 \mathrm{ng} / \mathrm{ml})$ and $10 \%$ FCS. Apoptotic cell death was induced via cytokine and serum depletion. Cell cultures were supplemented with ASPS $(100 \mu \mathrm{g} / \mathrm{ml})$, TPO $(100 \mathrm{ng} / \mathrm{ml})$ or LY294002 $(25 \mu \mathrm{M})$ and then cells were incubated at $37^{\circ} \mathrm{C}$ for $72 \mathrm{~h}$. Apoptotic cell death (percentage of early + late apoptotic cells) was evaluated using the Annexin V-FITC/PI, active caspase-3-PE and JC-1 ApoAlert reagent kits (BD Biosciences), as previously described $(18,20,21)$ or according to the manufacturer's instructions (for ApoAlert reagent kit). Apoptotic cells were grouped based on their percentages as 'early' (R2; $\mathrm{FITC}^{+}$and PI'), 'late' $\left(\mathrm{R} 1 ; \mathrm{FITC}^{+}\right.$and $\left.\mathrm{PI}^{+}\right)$and total $\left(\mathrm{R} 1+\mathrm{R} 2\right.$; $\left.\mathrm{FITC}^{+}\right)$apoptotic cells. A total of 10,000 events were acquired for each sample and were analyzed on the FACSCanto ${ }^{\mathrm{TM}}$ Flow Cytometry system (BD Biosciences) using the Lysis II C32 software (FACScan; BD Pharmingen; BD Biosciences) $(10,18,20)$.The dose of ASPS was chosen as previously reported (10).

Western blot analysis. For AKT and phosphorylated (p)-AKT immunodetection, cells were plated into 35-mm diameter plates at initial density of $5 \times 10^{5}$ cells, and were serum-starved overnight. When required, a 30-min pre-incubation step at $37^{\circ} \mathrm{C}$ with the PI3K inhibitor LY294002 (Sigma-Aldrich; Merck $\mathrm{KGaA}$ ) was performed prior to stimulation. Cells were stimulated for the indicated time-points at $37^{\circ} \mathrm{C}$ for $30 \mathrm{~min}$ with TPO $(100 \mathrm{ng} / \mathrm{ml})$ or ASPS $(100 \mu \mathrm{g} / \mathrm{ml})$. Subsequently, cells were rinsed rapidly in ice-cold PBS and lysed in 2\% SDS (Sigma-Aldrich; Merck KGaA) buffer containing $125 \mathrm{mM}$ Tris (pH 6.8). Lysates were then sonicated in an ice water bath for $2 \mathrm{~min}(5 \mathrm{sec}$ exposure separated by $10 \mathrm{sec}$ intervals), and the protein concentration was quantified using the DC Protein assay (Bio-Rad Laboratories, Inc). Cell lysates (40 $\mu \mathrm{g} /$ lane) were resolved using 8\% SDS-PAGE. Following protein transfer, membranes were blocked with 5\% non-fat dry milk in TBS-Tween-20 buffer, containing $20 \mathrm{mM}$ Tris- $\mathrm{HCl}$ (pH 7.4), $150 \mathrm{mM} \mathrm{NaCl}$ and $0.05 \%$ Tween-20, for $1 \mathrm{~h}$ at room temperature. Subsequently, membranes were probed with the appropriate primary antibodies (1:1,000; all from Santa Cruz Biotechnology, Inc.; $\beta$-actin, cat. no. sc-47778; AKT, cat. no. sc-5298; p-AKT, cat. no. sc-293125) at $4^{\circ} \mathrm{C}$ overnight. The next day membranes were incubated at room temperature for $1 \mathrm{~h}$ with a corresponding peroxidase-conjugated secondary antibody (1:1,000; anti-mouse IgGк; cat. no. sc-516102) at the dilutions recommended by the manufacturers. Blots were visualized using an ECL (Amersham; Cytiva) western blotting detection system (19).

Statistical analysis. Data are presented as the mean $\pm \operatorname{SD}(n=6)$. Statistical analysis was performed using one-way ANOVA, followed by Tukey's HSD test. $\mathrm{P}<0.05$ was considered to indicate a statistically significant difference. All statistical analyses were performed using the SPSS 19.0 software (IBM Corp.).

\section{Results}

Effects of ASPS on hematopoiesis in vitro. The results demonstrated that ASPS at 50, 100 and $200 \mu \mathrm{g} / \mathrm{ml}$ had a significant effect on the formation of CFU-GM and CFU-MK compared with $0 \mu \mathrm{g} / \mathrm{ml}$ group. ASPS at only $100 \mu \mathrm{g} / \mathrm{ml}$ had a significant effect on the formation of CFU-F. However, ASPS did not affect the formation of BFU/CFU-E (Fig. 1).

Effects of ASPS on a radiation-induced hemocytopenia/thrombocytopenia mouse model.

ASPS increases blood cell counts in the hemocytopenialthrombocytopenia mouse model. The changes in the counts of different blood cell subpopulations are presented in Fig. 2. The number of white blood cells (WBC) in each group was decreased to the lowest level on day 7 following exposure to irradiation and was gradually increased thereafter. 
A

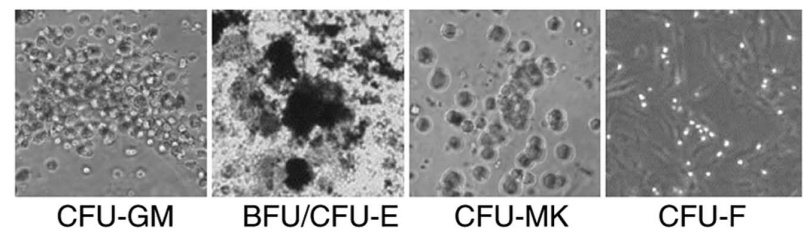

B

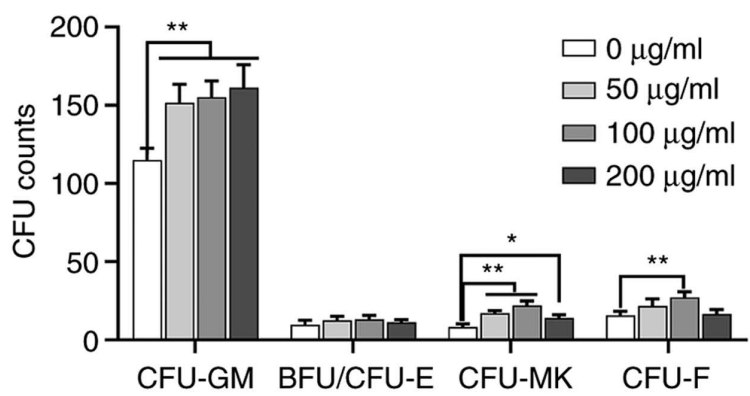

Figure 1. Effects of different concentrations of ASPS on the number of CFUs in vitro. (A) Representative image of CFU-GM, BFU/CFU-E, CFU-MK and CFU-F (magnification, x10). (B) Effect of 0, 50, 100 and $200 \mu \mathrm{g} / \mathrm{ml}$ ASPS on the formation of CFU-GM, BFU/CFU-E, CFU-MK and CFU-F. A concentration of $100 \mu \mathrm{g} / \mathrm{ml}$ ASPS exhibited the maximum effect. ${ }^{*} \mathrm{P}<0.05$, ${ }^{* *} \mathrm{P}<0.01$. ASPS, Astragalus polysaccharide; CFU-MK, colony-forming unit-megakaryocyte; CFU-GM, colony-forming unit-granulocyte macrophage; BFU/CFU-E, burst-forming unit/colony-forming unit-erythroid; CFU-GEMM, colony-forming unit- granulocyte, erythroid, monocyte and megakaryocyte; CFU-F, colony-forming unit-fibroblast.

On days 14 and 21, the WBC count in the ASPS-treated group was significantly higher compared with that to the control group. Similar results were observed in the WBC counts between the TPO-treated and control groups (Fig. 2A).

Following ASPS and TPO administration, the red blood cell (RBC) counts in the ASPS- and TPO-treated groups were elevated after day 7 (Fig. 2B). The RBC counts did not demonstrate a statistically significant difference between the ASPS-treated and control groups on day 7 and day 14. However, the RBC counts in the ASPS-treated group were significantly increased compared with the control group on day 21. There was no significant difference in the RBC counts between the TPO-treated and control groups from day 7 to day 21 .

The myelosuppressed mice exhibited a platelet nadir $\left(<300 \times 10^{9} / 1\right)$ on day 7 . Platelet counts were gradually increased from day 7 to day 21 (Fig. 2C). Following ASPS (12.5 mg/day) administration, the number of platelets was significantly elevated compared with the control group at day 14.

ASPS has no effect on the total body weight in the hemocytopenialthrombocytopenia mouse model. The total body weights in the control, ASPS- and TPO-treated groups were lowest on day 7 and were gradually increased thereafter. However, there was no statistically significant difference in the body weight of all mice in the ASPS-treated group compared with the control and TPO-treated groups on days 14 and 21 (Fig. 3).

ASPS significantlyenhances the CFU values of bone marrow in the hemocytopenialthrombocytopenia mouse model. Bone marrow cells in the ASPS-, TPO-treated and control mice were collected and cultured for CFU assays. Treatment with ASPS significantly increased the number of CFU-GM, BFU/CFU-E, CFU-GEMM, CFU-MK and CFU-F colonies (n=6; Fig. 4). Furthermore, TPO significantly increased the CFU counts in all cell lineages compared with the control group. It was found
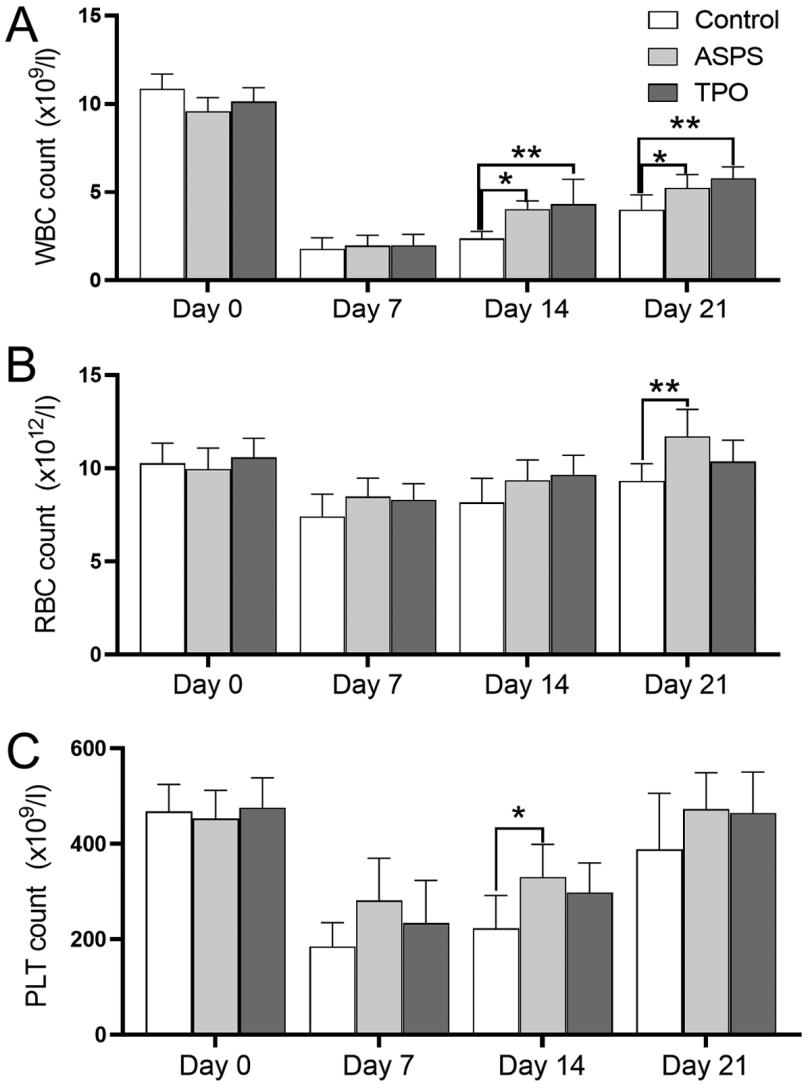

Figure 2. ASPS promotes the recovery of blood cells from irradiated mice. Mice $(n=6)$ were irradiated on day 0 and then treated with vehicle (control), ASPS (12.5 mg/day) and TPO (1 $\mu \mathrm{g} / \mathrm{kg} / \mathrm{day})$. Blood was collected and (A) WBC, (B) RBC and (C) PLTs were counted. Statistical analyses were performed between the cell counts of the corresponding and control groups (one-way ANOVA). ${ }^{*} \mathrm{P}<0.05,{ }^{* *} \mathrm{P}<0.01$. ASPS, Astragalus polysaccharide; TPO, thrombopoietin; WBC, white blood cells; RBC, red blood cells; PLT, platelets.

that treatment with ASPS and TPO exhibited similar effects in CFU-GM, CFU-GEMM and CFU-MK counts. Additionally, TPO had a much stronger effect on the number of BFU/CFU-E colonies compared with ASPS $(\mathrm{n}=6)$.

ASPS protects megakaryocytic cells in bone marrow. In the present study the morphology of bone marrow cells was also investigated using the Giemsa staining (Fig. 5). Compared with the control group, the tri-lineage hematopoiesis was preserved in the ASPS- and TPO-treated groups. This effect was particularly prominent in the megakaryocytic and granulocytic cells, but not in the erythroid one. The number of megakaryocytic and granulocytic cells was notable elevated in the ASPS- and TPO-treated groups, compared with that in the control group, indicating an improved recovery of these cells compared with those of the erythroid series. Furthermore, in the ASPS-treated group, overall cellularity (MTC/area) was similar to that in the TPO-treated group, mainly due to a prominent granulocytic expansion. Finally, the recovery of the megakaryocytes was markedly increased in the TPO-treated group compared with the control group by day 21 .

Effects of ASPS may be mediated via a TPO-independent pathway. To determine whether ASPS could alter the expression of TPO, ELISA was performed to measure the plasma concentration of TPO in different mice groups. There were no 


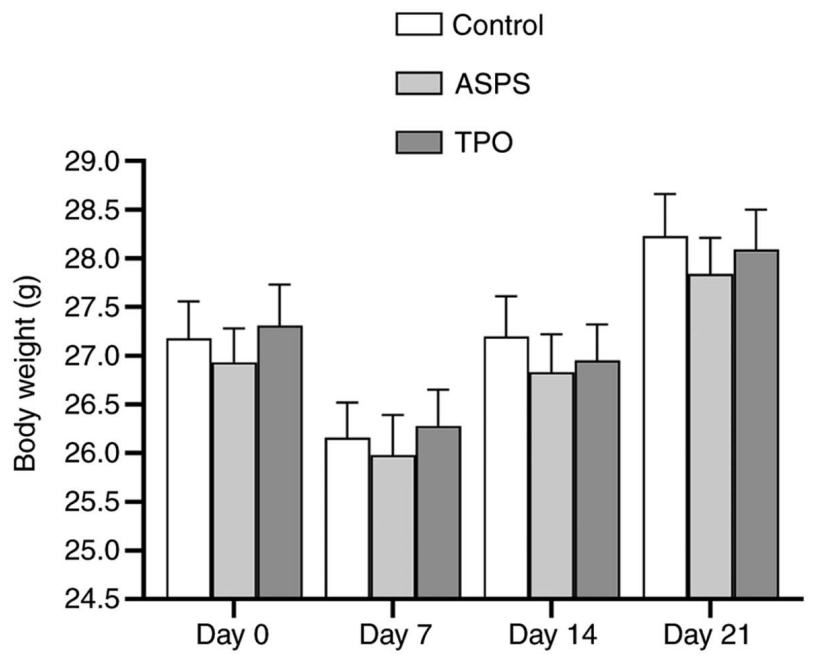

Figure 3. Effect of ASPS on the total body weight of irradiated mice. Statistical analyses (one-way ANOVA) were conducted between the body weight of mice treated with ASPS and TPO, and those in the control group $(n=6)$. ASPS, Astragalus polysaccharide; TPO, thrombopoietin.

significant differences in the plasma TPO levels between the normal, control and ASPS-treated mice (Fig. 6).

\section{Effects of ASPS on M-07e cells in vitro.}

ASPS attenuates apoptosis in M-07e cells. Cell apoptosis was evaluated in different treatment groups using the Annexin V assay (Fig. 7A). Apoptotic cells were grouped based on their percentages as 'early' (R2; $\mathrm{FITC}^{+}$and $\left.\mathrm{PI}^{-}\right)$, 'late' (R1; $\mathrm{FITC}^{+}$and $\left.\mathrm{PI}^{+}\right)$and total $\left(\mathrm{R} 1+\mathrm{R} 2 ; \mathrm{FITC}^{+}\right)$apoptotic cells. The lowest percentage of apoptotic cells was obtained in normal samples and the highest one in the LY294002-treated samples, whereas an intermediate percentage of apoptotic cells was observed in the control samples. Treatment with ASPS and TPO significantly decreased the early (R2), late (R1), and total (R1 + R2) apoptotic cells compared with the control group, and their effects were not significantly different between each other. Compared with treatment with LY294002 alone, co-treatment with ASPS decreased the percentage of late apoptotic cells (R1) from 27.75 to $15.88 \%$, early apoptotic (R2) from 18.18 to $13.10 \%$ and total apoptotic $(\mathrm{R} 1+\mathrm{R} 2)$ cells from 45.93 to $28.98 \%$.

The antiapoptotic effect of ASPS were assessed using the JC-1 assay, which is based on the calculation of the apoptotic cell populations according to the distribution of the JC-1 compound in aggregate and monomer form. In healthy cells, JC-1 aggregates were detected in mitochondria (Fig. 7B; red fluorescence; detected in FL2) by emitting red fluorescence, while the monomeric form was detected the cytoplasm. However, in apoptotic cells, JC-1 aggregates emitted green fluorescence due to the breakdown of the mitochondrial transmembrane potential (Fig. 7B; green fluorescence; detected in FL1). In Fig. 7B, R1 represents cells with both JC-1 aggregates and monomers, R2 cells with only JC-1 monomers and R1 + R2 represents the total number of apoptotic cells. The percentage of JC-1 monomer positive cells was significantly increased in the control, LY294002- and ASPS + LY294002-treated cells, compared with normal M-07e cells (Fig. 7B), indicating that the induction of apoptosis in these cells. Interestingly, no
A
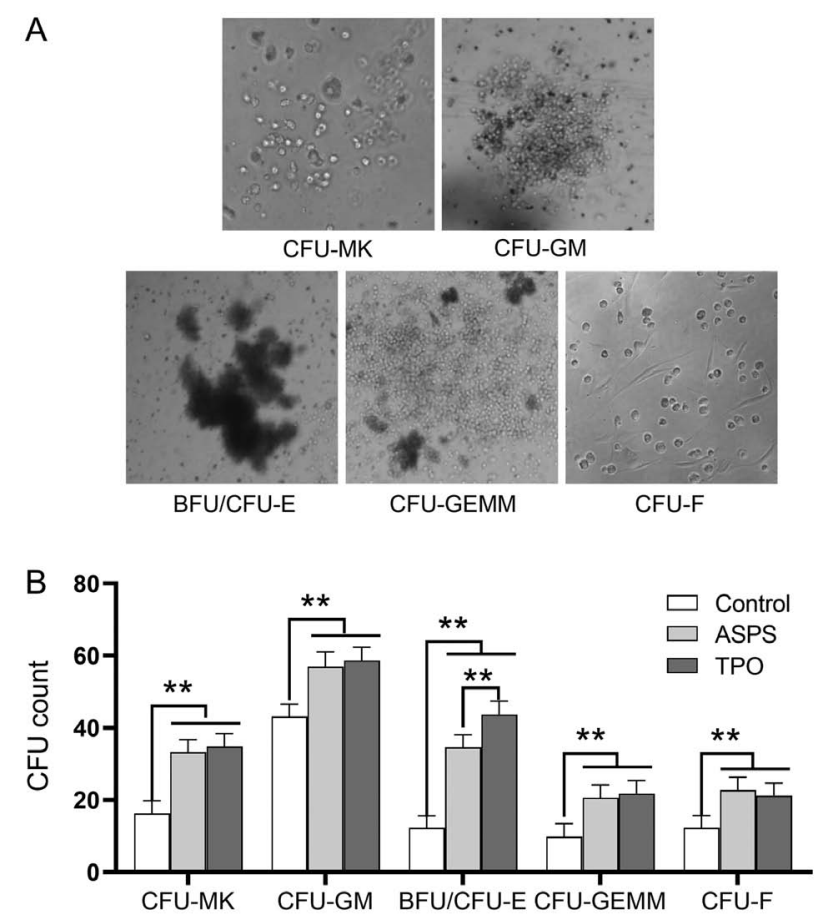

Figure 4. ASPS promotes the formation of CFU-GM, BFU/CFU-E, CFU-GEMM,CFU-MK and CFU-F cells in vivo. The colony counts for various $\mathrm{CFU}$, with or without treatment with ASPS are presented. (A) Representative image of CFU-MK, CFU-GM, BFU/CFU-E, CFU-GEMM and CFU-F (magnification, $x 10)$. (B) Statistical analyses were performed between the colony numbers of ASPS and TPO-treated samples, and those of the control samples (one-way ANOVA). ${ }^{* *} \mathrm{P}<0.01 ; \mathrm{n}=6$. ASPS, Astragalus polysaccharide; TPO, thrombopoietin; CFU-MK, colony-forming unit-megakaryocyte; CFU-GM, colony-forming unit-granulocyte macrophage; BFU/CFU-E, burst-forming unit/colony-forming unit-erythroid; CFU-GEMM, colony-forming unit-granulocyte, erythroid, monocyte and megakaryocyte; CFU-F, colony-forming unit-fibroblast.

significant differences were observed in the percentage of apoptotic cells between the ASPS or TPO-treated samples and normal ones, suggesting that ASPS and TPO mediated protective effects against apoptosis.

Activation of caspase-3, a downstream effector protein of apoptosis, is indicative of apoptotic occurrence (22). In the caspase-3 assay, M-07e cells were treated as aforementioned, labelled with activated caspase-3-PE dye and subjected to flow cytometric analysis. Histograms and statistical analyses between the indicated and normal samples are presented in Fig. 7C. The percentage of activated caspase-3 positive cells in the ASPS- and TPO-treated cells was significantly decreased compared with the control group. In addition, ASPS- and TPO-treated cells were similar compared with in the normal samples. LY294002-treated, ASPS+LY294002-treated and control cells exhibited a significantly higher percentage of activated caspase-3 positive cells compared with normal cells.

ASPS attenuates apoptosis via activating the PI3K/AKT pathway. As indicated in Fig. 7, the percentage of apoptotic cells in ASPS + LY294002-treated cells was significantly higher compared with ASPS-treated cells. To confirm that ASPS inhibited cell apoptosis via the AKT pathway, the activation status of p-AKT was detected via western blot analysis (Fig. 8). p-AKT was detectable 15 min following treatment with ASPS. However, there was no obvious change in the phosphorylation levels of AKT at 30 and 60 min (Fig. 8A). Compared with 


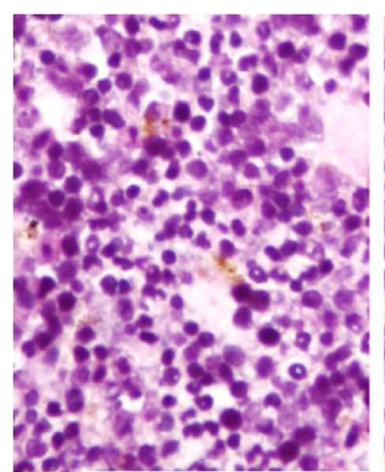

Control

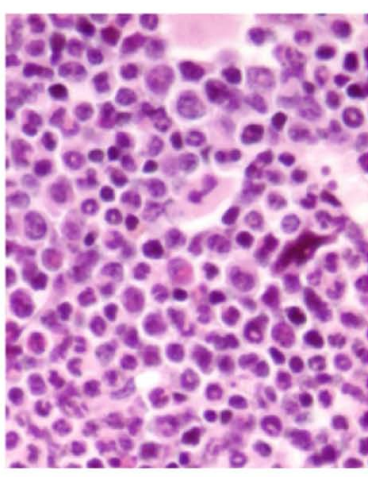

ASPS

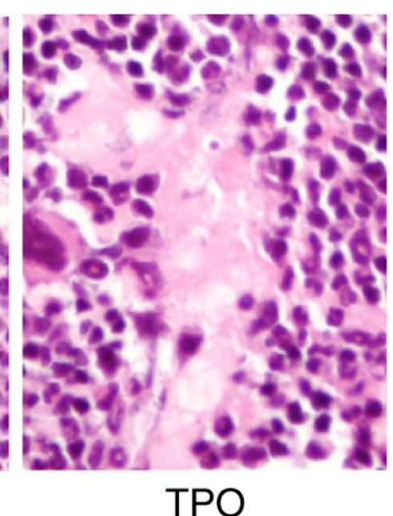

Figure 5. Effect of ASPS on bone marrow morphology (magnification, x400). Thrombopoiesis was preserved in the ASPS- and TPO-treated groups, compared with the control group. In both TPO- and ASPS-treated groups, a notable increased recovery of the megakaryocytic and granulocytic series by day 21 was observed, compared with the control group. ASPS, Astragalus polysaccharide; TPO, thrombopoietin.

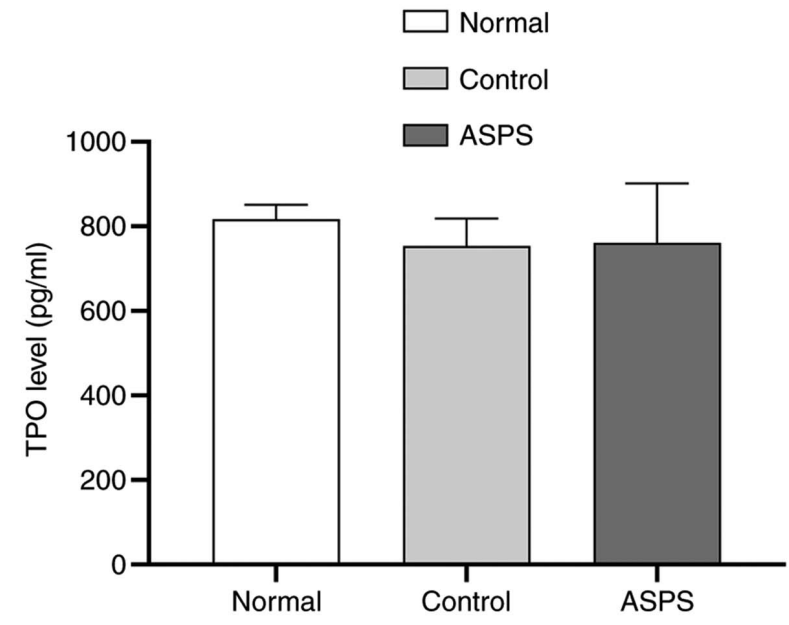

Figure 6. ASPS treatment had no effect on TPO levels. The levels of TPO in the normal, control and ASPS-treated samples were measured using ELISA. The differences among TPO levels were not statistically significant $(n=6)$. ASPS, Astragalus polysaccharide; TPO, thrombopoietin.

the control group, ASPS- and TPO-treated groups exhibited markedly increased AKT activation levels, whereas no visible difference between ASPS and TPO was observed. Moreover, treatment of M-07e cells with ASPS + LY294002 notably suppressed the ASPS-induced AKT activation (Fig. 8B).

\section{Discussion}

The present study demonstrated that ASPS enhanced the in vivo recovery of peripheral blood cells and platelets, in a murine model of hemocytopenia/thrombocytopenia. This effect was supported by the CFU analysis results, where treatment with ASPS stimulated the formation of CFU-MK and CFU-GEMM. CFU is an indicator for hematopoietic growth function of bone marrow. In the present experiment, bone marrow cells were treated with 0, 50, 100 and $200 \mu \mathrm{g} / \mathrm{ml}$ ASPS. It was identified that $100 \mu \mathrm{g} / \mathrm{ml}$ ASPS had maximum effect, and when exceeding this dose, the effect was gradually declined. In addition, ASPS inhibited megakaryocyte apoptosis. In summary, the current study supplemented our previous study on the mechanism of DBT by investigating the hematopoietic/thrombopoietic effects of ASPS $(10,11)$. However, in addition to the anti-apoptotic mechanism, other mechanisms of ASPS on hematopoiesis require further study.

Cell density is a classic indicator for evaluating the ability of retaining the hematopoietic phenotype in three-line cells, and has been widely used in previous articles $(18,23,24)$. The present results suggested that the platelet counts in ASPS-treated mice were significantly increased at an earlier or similar time-point compared with TPO-treated mice. In addition, ASPS exhibited a weak effect on the proliferation of WBCs and RBCs at day 14 and day 21. No significant difference was observed in WBC and RBC counts between the ASPS- and TPO-treated groups. These findings indicated that the radioprotective effect of ASPS in the recovery of peripheral blood cells was similar to that of TPO, especially in the recovery of platelets. Therefore, this study suggested that ASPS could be used as an alternative approach for the management of thrombocytopenia.

As a potential substitute of TPO, the thrombopoietic effect of ASPS by promoting the production of TPO remains unknown. He et al (25) reported that ASPS could inhibit TNF- $\alpha$ and IL-1 $\beta$ mRNA expression levels. Furthermore, it has been revealed that ASPS significantly suppresses NF- $\mathrm{B}$ activation (26), as well as downregulates the phosphorylation of ERK and JNK (27), two important signaling pathways involved in the expression of TNF- $\alpha$ and IL-1 $\beta$. The current study suggested that ASPS could increase the number of platelets in an inflammatory factor-independent manner. Our previous study demonstrated that IL- $1 \beta$ could upregulate TPO expression, thus resulting in the proliferation of platelets (28). Taken together, these findings, combined with the results of the present study, indicated that ASPS could promote the proliferation of megakaryocytes in a TPO-independent manner.

In our previous studies, M-07e and HL-60 cells were treated with $0,50,100$ and $200 \mu \mathrm{g} / \mathrm{ml} \mathrm{ASPS}$, and it was identified that $100 \mu \mathrm{g} / \mathrm{ml}$ had a higher stimulatory effect on cell proliferation $(29,30)$. In the present study, the antiapoptotic effect of ASPS on M-07e cells was demonstrated by performing Annexin V and activated caspase-3 assays $(18,20)$. The Annexin V and caspase-3 assay results demonstrated that the percentage of the apoptotic M-07e cells in the 
A

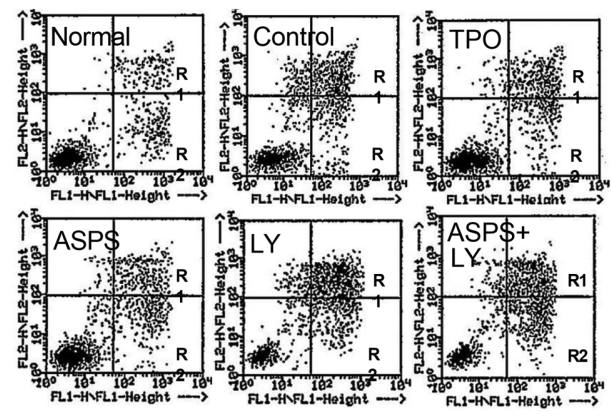

B
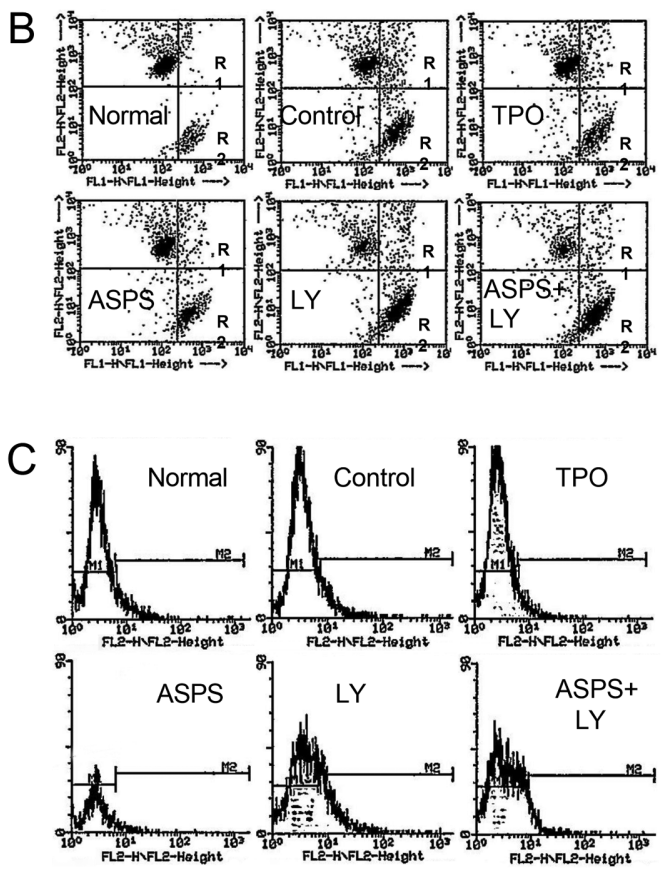
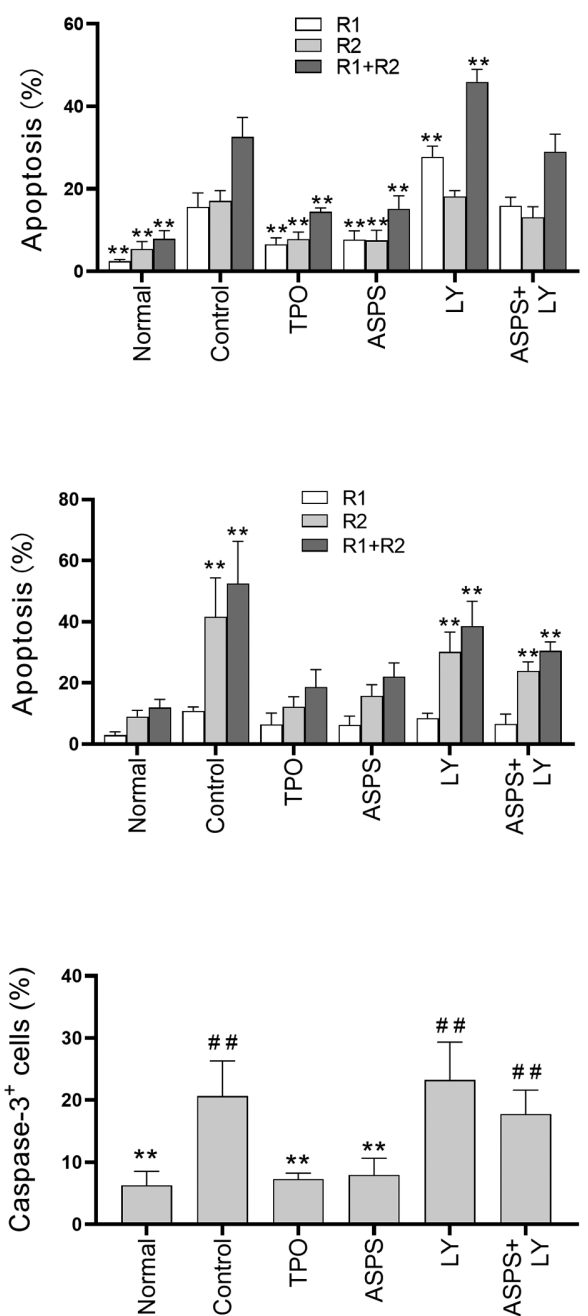

Figure 7. Effects of ASPS on cell apoptosis analyzed using Annexin V, JC-1 and caspase-3 assays. Apoptosis was induced via serum depletion (control). Cells were treated with ASPS, TPO or LY for $72 \mathrm{~h}$. (A) Dot plots, quantification and statistical analyses under various treatments were performed using the Annexin V assay. ${ }^{* *} \mathrm{P}<0.01$ vs. control group. (B) Dot plots, quantification and statistical analyses under various treatments were conducted using the JC-1 assay. ${ }^{* *} \mathrm{P}<0.01$ vs. normal group. (C) Dot plots, quantification and statistical analyses under various treatments were performed using the caspase-3 assay. R1, early apoptotic cells; R2, late apoptotic cells; $\mathrm{R} 1+\mathrm{R} 2$, total apoptotic cells. ${ }^{* *} \mathrm{P}<0.01$ vs. control group; ${ }^{\# \#} \mathrm{P}<0.01$ vs. normal group ( $\mathrm{n}=4$ ). ASPS, Astragalus polysaccharide; TPO, thrombopoietin; LY, LY294002.
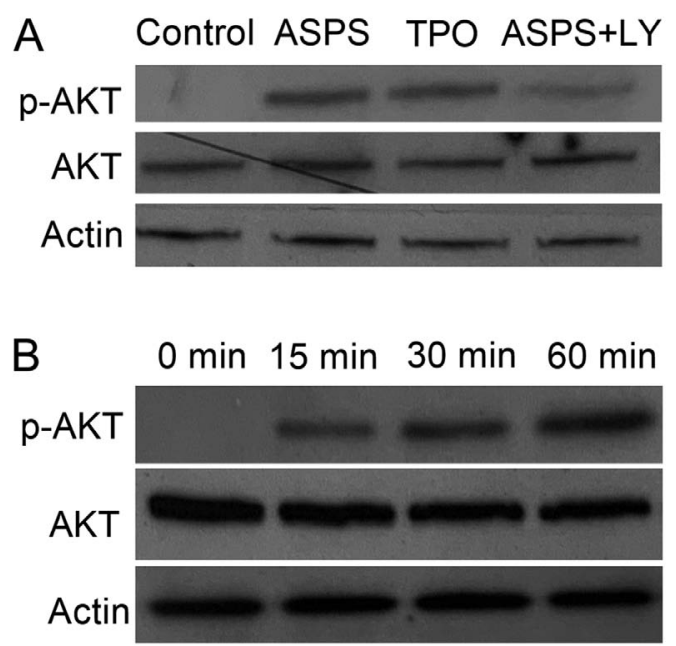

Figure 8. ASPS activates the PI3K/AKT pathway. p-AKT expression was detected via western blot analysis. (A) Protein levels of AKT and p-AKT in control, ASPS-, TPO- and TPO + LY294002-treated groups. (B) Protein expression levels of AKT and p-AKT at different time intervals. ASPS, Astragalus polysaccharide; $\mathrm{p}-$, phosphorylated; TPO, thrombopoietin.
ASPS-treated group was significantly decreased compared with that in the control samples (cytokine- and serum-depleted samples). With respect to the JC-1 distribution, the number of apoptotic cells in the control samples was significantly higher compared with that in the ASPS-treated and normal samples. These finding indicated that ASPS could enhance peripheral blood cell proliferation via inhibiting cell apoptosis, but not by directly stimulating bone marrow. Furthermore, the activation of caspase- 3 in the control samples was elevated compared with that in the ASPS-treated samples, suggesting that ASPS attenuated megakaryocyte apoptosis via mitochondrial-mediated pathways.

The PI3K/AKT pathway serves an important role in cell apoptosis (31) and proliferation (32) in a large spectrum of cell types, including megakaryocytes (33). Several studies have reported that cell apoptosis may be induced by regulating a variety of downstream effector molecules of the PI3K/AKT pathway $(34,35)$. As the key molecule of this pathway, p-AKT actively inhibits cell apoptosis (36). Therefore, decreased $\mathrm{p}$-AKT levels may increase the apoptotic rate of $\mathrm{M}-07 \mathrm{e}$ 
cells. TPO is a growth factor not only for platelets, but also for hematopoietic stem cells (37). TPO has been shown to inhibit apoptosis via activating the PI3K pathway in previous studies $(38,39)$, and it is a common drug for positive control in the study of hematopoiesis and thrombopoiesis $(13,40,41)$. The results of the present study indicated that ASPS inhibited $\mathrm{M}-07 \mathrm{e}$ cell apoptosis via regulating the levels of $\mathrm{p}-\mathrm{AKT}$ within the PI3K/AKT pathway.

In conclusion, in the present study, the hematopoietic/thrombopoietic effects of ASPS were confirmed in vivo and in vitro, which were attributed to two different mechanisms. Firstly, ASPS promoted the proliferation of hematopoietic CFUs, and secondly, inhibited megakaryocyte apoptosis. These findings may facilitate the development of novel alternative therapies for patients with myelosuppression, including thrombocytopenia.

\section{Acknowledgements}

The authors would like to thank Mr. Nga Hin Pong from The Chinese University of Hong Kong for technical assistance in the animal procedures and other studies.

\section{Funding}

This work was supported by the National Natural Science Foundation of China (grant no. 81770116), the Funding of Traditional Medicine Project from the Health Department of Guangming District, the Shenzhen, Science, Technology and Innovation Commission of Shenzhen, the China's Postdoctoral Science Foundation (grant no. 2019TQ0383) and the Sanming Project of Medicine in Shenzhen (grant no. SZSM202011004).

\section{Availability of data and materials}

The datasets used and/or analyzed during the current study are available from the corresponding author on reasonable request.

\section{Authors' contributions}

MY and LL contributed to the conception and design of the present study, and provided the final proofs of the manuscript version to be published. WX and LL conducted the experiments and collected data. LL, CKL, CY and CC performed the data and statistical analyses, and drafted the manuscript. MY, HXi, YC, CC, CKL, XF, LY and HXu analyzed the data and revised the manuscript repeatedly. All authors read and approved the final manuscript.

\section{Ethics approval and consent to participate}

The experimental protocol of the present study was approved by the Animal Research Welfare Committee of Southern Medical University. The principles of the National Institutes of Health Guidelines for Laboratory Animals were followed during the entire course of the experiments. All parts of this report are in compliance with the ARRIVE Guidelines for reporting animal research (42).

\section{Patient consent for publication}

Not applicable

\section{Competing interests}

The authors declare that they have no competing interests.

\section{References}

1. Liebman HA: Thrombocytopenia in cancer patients. Thromb Res 133 (Suppl 2): S63-S69, 2014.

2. Lieberman L, Bercovitz RS, Sholapur NS, Heddle NM Stanworth SJ and Arnold DM: Platelet transfusions for critically ill patients with thrombocytopenia. Blood 123: 1146-1151, quiz 1280, 2014.

3. Cines DB, Gernsheimer T, Wasser J, Godeau B, Provan D, Lyons R, Altomare I, Wang X and Lopez A: Integrated analysis of long-term safety in patients with chronic immune thrombocytopaenia (ITP) treated with the thrombopoietin (TPO) receptor agonist romiplostim. Int J Hematol 102: 259-270, 2015.

4. Arnold DM, Vrbensky JR, Karim N, Smith JW, Liu Y, Ivetic N, Kelton JG and Nazy I: The effect of rituximab on anti-platelet autoantibody levels in patients with immune thrombocytopenia. Br J Haematol 178: 302-307, 2017.

5. Aubron C, Flint AW, Bailey M, Pilcher D, Cheng AC, Hegarty C, Martinelli A, Reade MC, Bellomo R and McQuilten Z: Is platelet transfusion associated with hospital-acquired infections in critically ill patients? Crit Care 21: 2, 2017.

6. Scheinberg P, Singulane CC, Barbosa LS and Scheinberg M: Successful platelet count recovery in lupus-associated thrombocytopenia with the thrombopoietin agonist eltrombopag. Clin Rheumatol 33: 1347-1349, 2014.

7. Shin SK, Pack SP, Oh JG, Kang NK, Chang MH, Chung YH, Kim SJ, Lee JW and Heo TH: Anti-erythropoietin and antithrombopoietin antibodies induced after administration of recombinant human erythropoietin. Int Immunopharmacol 11: 2237-2241, 2011.

8. Gong AG, Li N, Lau KM, Lee PS, Yan L, Xu ML, Lam CT, Kong AY, Lin HQ, Dong TT, et al: Calycosin orchestrates the functions of Danggui Buxue Tang, a Chinese herbal decoction composing of Astragali Radix and Angelica Sinensis Radix: An evaluation by using calycosin-knock out herbal extract. J Ethnopharmacol 168: 150-157, 2015.

9. Lin HQ, Gong AG, Wang HY, Duan R, Dong TT, Zhao KJ and Tsim KW: Danggui Buxue Tang (Astragali Radix and Angelicae Sinensis Radix) for menopausal symptoms: A review. J Ethnopharmacol 199: 205-210, 2017.

10. Liu C, Li J, Meng FY, Liang SX, Deng R, Li CK, Pong NH, Lau CP, Cheng SW, Ye JY, et al: Polysaccharides from the root of Angelica sinensis promotes hematopoiesis and thrombopoiesis through the PI3K/AKT pathway. BMC Complement Altern Med 10: 79, 2010.

11. Yang M, Chan GC, Deng R, Ng MH, Cheng SW, Lau CP, Ye JY, Wang L and Liu C: An herbal decoction of Radix astragali and Radix angelicae sinensis promotes hematopoiesis and thrombopoiesis. J Ethnopharmacol 124: 87-97, 2009.

12. Zhao M, Zhang ZF, Ding Y, Wang JB and Li Y: Astragalus polysaccharide improves palmitate-induced insulin resistance by inhibiting PTP1B and NF- $\mathrm{BB}$ in $\mathrm{C}_{2} \mathrm{Cl}_{2}$ myotubes. Molecules 17: 7083-7092, 2012.

13. Xie JH, Jin ML, Morris GA, Zha XQ, Chen HQ, Yi Y, Li JE, Wang ZJ, Gao J, Nie SP, et al: Advances on bioactive polysaccharides from medicinal plants. Crit Rev Food Sci Nutr 56 (Suppl 1): S60-S84, 2016.

14. National Research Council: Guide for the Care and Use of Laboratory Animals. National Academies Press, Washington, DC, 1985.

15. Yuan S, Piao X, Li D, Kim S, Lee H and Guo P: Effects of dietary Astragalus polysaccharide on growth performance and immune function in weaned pigs. Anim Sci 82: 501-507, 2006.

16. Staub A: Removeal of protein-Sevag method. Methods Carbohydr Chem 5: 5-6, 1965.

17. Inagaki K, Oda T, Naka Y, Shinkai H, Komatsu N and Iwamura H: Induction of megakaryocytopoiesis and thrombocytopoiesis by JTZ-132, a novel small molecule with thrombopoietin mimetic activities. Blood 104: 58-64, 2004. 
18. Ye JY, Chan GC, Qiao L, Lian Q, Meng FY, Luo XQ Khachigian LM, Ma M, Deng R, Chen JL, et al: Platelet-derived growth factor enhances platelet recovery in a murine model of radiation-induced thrombocytopenia and reduces apoptosis in megakaryocytes via its receptors and the PI3-k/Akt pathway. Haematologica 95: 1745-1753, 2010.

19. Yang M, Li K, Chui CM, Yuen PM, Chan PK, Chuen CK, Li CK and Fok TF: Expression of interleukin (IL) 1 type I and type II receptors in megakaryocytic cells and enhancing effects of IL-1beta on megakaryocytopoiesis and NF-E2 expression. Br J Haematol 111: 371-380, 2000.

20. Yang M, Li K, Ng PC, Chuen CK, Lau TK, Cheng YS, Liu YS, Li CK, Yuen PM, James AE, et al: Promoting effects of serotonin on hematopoiesis: Ex vivo expansion of cord blood CD34 stem/progenitor cells, proliferation of bone marrow stromal cells, and antiapoptosis. Stem Cells 25: 1800-1806, 2007.

21. Li K, Sung RY, Huang WZ, Yang M, Pong NH, Lee SM, Chan WY, Zhao H, To MY, Fok TF, et al: Thrombopoietin protects against in vitro and in vivo cardiotoxicity induced by doxorubicin. Circulation 113: 2211-2220, 2006

22. Porter AG and Jänicke RU: Emerging roles of caspase-3 in apoptosis. Cell Death Differ 6: 99-104, 1999.

23. Zhang J, Zhou S, Zhou Y, Feng F, Wang Q, Zhu X, Zhao J, $\mathrm{Fu} \mathrm{H}, \mathrm{Lv} \mathrm{M}, \mathrm{Ai} \mathrm{H}$, et al: Adipose-derived mesenchymal stem cells (ADSCs) with the potential to ameliorate platelet recovery, enhance megakaryopoiesis, and inhibit apoptosis of bone marrow cells in a mouse model of radiation-induced thrombocytopenia Cell Transplant 25: 261-273, 2016.

24. MacVittie TJ, Farese AM, Smith WG, Baum CM, Burton E and McKearn JP: Myelopoietin, an engineered chimeric IL-3 and G-CSF receptor agonist, stimulates multilineage hematopoietic recovery in a nonhuman primate model of radiation-induced myelosuppression. Blood 95: 837-845, 2000

25. He X, Shu J, Xu L, Lu C and Lu A: Inhibitory effect of Astragalus polysaccharides on lipopolysaccharide-induced TNF- $\alpha$ and IL-1 $\beta$ production in THP-1 cells. Molecules 17: 3155-3164, 2012.

26. Xue H, Gan F, Zhang Z, Hu J, Chen X and Huang K: Astragalus polysaccharides inhibits PCV2 replication by inhibiting oxidative stress and blocking NF- $\kappa \mathrm{B}$ pathway. Int J Biol Macromol 81: 22-30, 2015.

27. Han L and Wang HX and Lu MI: Effect of Astragalus polysaccharide on LPS-induced cardiomyocyte apoptosis by inhibiting $\mathrm{NF}-\kappa \mathrm{B}$ and JNK signaling pathway. Chinese Pharmacol Bull 34 243-249, 2018.

28. Chuen CK, Li K, Yang M, Fok TF, Li CK, Chui CM and Yuen PM: Interleukin-1 $\beta$ up-regulates the expression of thrombopoietin and transcription factors c-Jun, c-Fos, GATA-1, and NF-E2 in megakaryocytic cells. J Lab Clin Med 143: 75-88, 2004.

29. Xiao B, Xu Y, He H, Jiang QL, Li SY, Shu HY, Liang EY, Yi ZS, Ye JY, Huang LF, et al: Anti-apoptotic effect of Astragalus Polysaccharide on myeloid cells. Zhongguo Shi Yan Xue Ye Xue Za Zhi 21: 1243-1247, 2013 (In Chinese).

30. Yang M, Jiang Q, Xiao B, Liu C, Li S, Huang L, Chong B and Meng F: Astragalus polysaccharide has hematopoietic and thrombopoietic activities in an irradiation mouse model. Blood 122 $4216,2013$.
31. Jiang X, Zeng L, Huang J, Zhou H and Liu Y: Arctigenin, a natural lignan compound, induces apoptotic death of hepatocellular carcinoma cells via suppression of PI3-K/Akt signaling. J Biochem Mol Toxicol 29: 458-464, 2015.

32. Liu R, Ding L, Yu MH, Wang HQ, Li WC, Cao Z, Zhang P, Yao BC, Tang J, Ke Q, et al: Effects of dihydrotestosterone on adhesion and proliferation via PI3-K/Akt signaling in endothelial progenitor cells. Endocrine 46: 634-643, 2014.

33. Martini M, De Santis MC, Braccini L, Gulluni F and Hirsch E: PI3K/AKT signaling pathway and cancer: An updated review. Ann Med 46: 372-383, 2014.

34. $\mathrm{Hu} \mathrm{C}, \mathrm{Xu} \mathrm{M}$, Qin R, Chen $\mathrm{W}$ and $\mathrm{Xu} \mathrm{X}$ : Wogonin induces apoptosis and endoplasmic reticulum stress in HL-60 leukemia cells through inhibition of the PI3K-AKT signaling pathway. Oncol Rep 33: 3146-3154, 2015.

35. Ma W, Wang DD, Li L, Feng YK, Gu HM, Zhu GM, Piao JH, Yang Y, Gao X and Zhang PX: Caveolin-1 plays a key role in the oleanolic acid-induced apoptosis of HL-60 cells. Oncol Rep 32: 293-301, 2014.

36. Liu Y, Bi T, Wang G, Dai W, Wu G, Qian L, Gao Q and Shen G: Lupeol inhibits proliferation and induces apoptosis of human pancreatic cancer PCNA-1 cells through AKT/ERK pathways. Naunyn Schmiedebergs Arch Pharmacol 388: 295-304, 2015.

37. Hitchcock IS and Kaushansky K: Thrombopoietin from beginning to end. Br J Haematol 165: 259-268, 2014.

38. Pulikkan JA, Madera D, Xue L, Bradley P, Landrette SF, Kuo YH, Abbas S, Zhu LJ, Valk P and Castilla LH: Thrombopoietin/MPL participates in initiating and maintaining RUNX1-ETO acute myeloid leukemia via PI3K/AKT signaling. Blood 120: 868-879, 2012.

39. Chan KY, Xiang P, Zhou L, Li K, Ng PC, Wang CC, Zhang L, Deng HY, Pong NH, Zhao H, et al: Thrombopoietin protects against doxorubicin-induced cardiomyopathy, improves cardiac function, and reversely alters specific signalling networks. Eur J Heart Fail 13: 366-376, 2011.

40. Su RJ,Zhang XB, Li K, Yang M, Li CK, Fok TF, James AE, Pong H and Yuen PM: Platelet-derived growth factor promotes ex vivo expansion of $\mathrm{CD}_{3} 4^{+}$cells from human cord blood and enhances long-term culture-initiating cells, non-obese diabetic/severe combined immunodeficient repopulating cells and formation of adherent cells. Br J Haematol 117: 735-746, 2002.

41. Ye JY, Liang EY, Cheng YS, Chan GC, Ding Y, Meng F, Ng MH, Chong BH, Lian Q and Yang M: Serotonin enhances megakaryopoiesis and proplatelet formation via p-Erk1/2 and F-actin reorganization. Stem Cells 32: 2973-2982, 2014.

42. Kilkenny C, Browne WJ, Cuthill IC, Emerson M and Altman DG: Improving bioscience research reporting: The ARRIVE guidelines for reporting animal research. J Pharmacol Pharmacother 1: 94-99, 2010. 